\title{
Time-varying uncertainty in shock and vibration applications using the impulse response
}

\author{
J.B. Weathers ${ }^{\mathrm{a}, *}$ and Rogelio Luck ${ }^{\mathrm{b}}$ \\ ${ }^{a}$ Shock, Noise, and Vibration Group, Northrop Grumman Shipbuilding, Pascagoula, MS, USA \\ ${ }^{\mathrm{b}}$ Department of Mechanical Engineering, Mississippi State University, Mississippi State, MS, USA
}

Received 3 November 2010

Revised 21 February 2011

\begin{abstract}
Design of mechanical systems often necessitates the use of dynamic simulations to calculate the displacements (and their derivatives) of the bodies in a system as a function of time in response to dynamic inputs. These types of simulations are especially prevalent in the shock and vibration community where simulations associated with models having complex inputs are routine. If the forcing functions as well as the parameters used in these simulations are subject to uncertainties, then these uncertainties will propagate through the models resulting in uncertainties in the outputs of interest. The uncertainty analysis procedure for these kinds of time-varying problems can be challenging, and in many instances, explicit data reduction equations (DRE's), i.e., analytical formulas, are not available because the outputs of interest are obtained from complex simulation software, e.g. FEA programs. Moreover, uncertainty propagation in systems modeled using nonlinear differential equations can prove to be difficult to analyze. However, if (1) the uncertainties propagate through the models in a linear manner, obeying the principle of superposition, then the complexity of the problem can be significantly simplified. If in addition, (2) the uncertainty in the model parameters do not change during the simulation and the manner in which the outputs of interest respond to small perturbations in the external input forces is not dependent on when the perturbations are applied, then the number of calculations required can be greatly reduced. Conditions (1) and (2) characterize a Linear Time Invariant (LTI) uncertainty model. This paper seeks to explain one possible approach to obtain the uncertainty results based on these assumptions.
\end{abstract}

Keywords: Uncertainty, sensitivity analysis, model simulation, transient uncertainty

\section{Introduction}

The uncertainty analysis of time-varying simulation results presents challenges not typically faced when conducting routine detailed uncertainty analyses of steady-state results. Well-accepted methodologies exist for calculating the uncertainty associated with steady-state results; however, methodologies which can be used to efficiently deal with uncertainties associated with time-varying or transient simulation results are limited at best [1,2,11-14]. For numerous types of transient engineering problems, countless time increments are needed to accurately resolve the output(s) of interest. Therefore, methods to efficiently perform the uncertainty calculations associated with each time increment is of primary interest. This dilemma is compounded by the fact that large complex models with extensive simulation runtimes have become common place in the engineering community making performing many simulation runs extremely cumbersome. Though ever-increasing computational power assists in this predicament, many times, performing hundreds and sometimes thousands of simulation runs is not feasible under strict program objectives.

\footnotetext{
${ }^{*}$ Corresponding author. Tel.: +1 662312 9017; E-mail: jamesweathers9@gmail.com.
} 
Thus, brute force methods such as the traditional Monte Carlo analysis are impractical for these applications. In addition, often the simulation uncertainty is only one part of the exercise which may include a comparison to benchmark results at similar conditions (i.e. a validation exercise). This possibility only increases the need to expedite simulation uncertainty calculations.

One way to handle time-varying uncertainties associated with LTI engineering applications is by taking advantage of the well-accepted mathematics related to the response of a linear system to an arbitrary excitation. This theory is commonly used in the shock and vibration community while dealing with response spectrum analysis or shock response spectrums (SRS). Assuming a LTI uncertainty model using the principle of superposition, one can derive the equation for the LTI system response to any arbitrary excitation with the help of the impulse response through a convolution process [4]. This approach can then be used to derive an efficient way to calculate time-varying uncertainties associated with simulation results. As previously stated, this methodology applies to results with uncertainties that propagate in a linear, time invariant manner. In other words, the uncertainties associated with the simulation/experimental results are assumed to obey the superposition principle, and the simulation response to input perturbations is assumed to be independent of when the perturbations are applied. Relatively simple exercises can be used to test these conditions. Furthermore, these assumptions are not to be confused with a response which is a linear function of the model arguments (i.e. model parameters, external inputs, etc....). Even for highly nonlinear problems, a linear uncertainty model may prove to be a valid assumption depending heavily on the magnitude of the uncertainties in the model arguments. For simulation results which are nonlinear with respect to the model arguments, the likelihood that the model argument sensitivities will remain constant within these uncertainty intervals decreases as the magnitude of these intervals increases [7]. Furthermore, in the Authors' experience, linear uncertainty models are extremely common in the engineering community over a wide spectrum of disciplines including shock and vibration. Increasing the knowledge associated with your model arguments can often times drastically decrease these uncertainties thereby increasing the chances of having a linear uncertainty model.

Since most simulation results are sampled in time, a simulation typically yields a total of $M$ results collected at discrete time intervals, $t_{1}, t_{2}, \ldots, t_{M}$. For many applications, the result of a numerical simulation will depend on the following input information:

a) the set of model parameters $\left\{\bar{a}_{1}, \bar{a}_{2}, \ldots, \bar{a}_{p}\right\}$

b) the set of initial conditions/final conditions $\left\{\bar{c}_{1}, \bar{c}_{2}, \ldots, \bar{c}_{q}\right\}$

c) and the complete time history of discretized external input(s) $\left\{\bar{g}\left(t_{1}\right), \bar{g}\left(t_{2}\right), \ldots, \bar{g}\left(t_{N}\right)\right\}$

The functional relationship for the result at the $k$-th time step may be expressed as

$$
R_{k}=R\left(t_{k}\right)=f_{k}\left(\bar{a}_{1}, \bar{a}_{2}, \ldots, \bar{a}_{p}, \bar{c}_{1}, \bar{c}_{2}, \ldots, \bar{c}_{q}, \bar{g}\left(t_{1}\right), \bar{g}\left(t_{2}\right), \ldots, \bar{g}\left(t_{N}\right)\right) k=1,2, \ldots, M
$$

Note that the functions $f_{k}(\cdot)$, for $k=1,2, \ldots, M$, are different for each time step $k$. To elucidate the chosen notation in Eq. (1), consider a system that is linear with respect to the external inputs. In such a case, the results can be expressed as $\{R\}=[F] \cdot\{g\}$, i.e., a matrix $[F]$ multiplying a vector $\{g\}$ of discretized external inputs. This means that $f_{k}$ is obtained by the dot product between the $k$-th row of matrix $[F]$ and the vector $\{g\}$ of external inputs. If in addition, the system is known to be causal, i.e., only external inputs from the past can affect the future, then $[F]$ will be lower diagonal.

In the following sections, examples are presented to show how to obtain the sensitivities of the result $R_{k}$ to the arguments shown in Eq. (1). The first example consists of a rather simplistic SDOF mass-spring-damper system used to explain the details of the uncertainty analysis methodology without dwelling on the complexities of a more sophisticated problem. The second example illustrates the use of the transient uncertainty analysis methodology in a more realistic and complex time-varying problem involving the finite element analysis (FEA) of a double isolated air compressor.

\section{Derivation of methodology using SDOF system}

Let us consider the single degree of freedom (SDOF) mass-spring-damper system presented in Fig. 1. The differential equation for the system illustrated in Fig. 1 is presented as Eq. (2). 


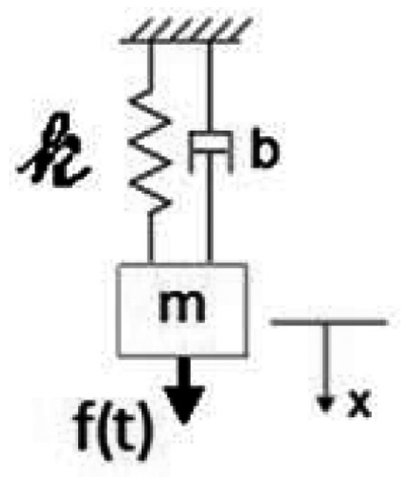

Fig. 1. Mass-spring-damper system.

$$
m \ddot{x}+b \dot{x}+k x=f(t)
$$

The model parameters associated with this problem are the spring stiffness $(k)$, the damping coefficient $(b)$, and the mass $(m)$. The initial conditions are the initial position $x_{0}$ and the initial velocity $v_{0}$. The external input corresponds to the force $f(t)$, and the calculation result or output of interest is the displacement of the mass, $x(t)$. The coordinate system is assumed to start at the static equilibrium position so the gravitational constant can be omitted.

In this example, one would expect as many calculated displacement values as external input force values, implying that $N=M$. The equation for the $k$-th calculated result $R_{k}$ can then be expressed as:

$$
R_{k}=x_{k}\left(\bar{k}, \bar{b}, \bar{m}, \bar{x}_{0}, \bar{v}_{0}, \bar{f}\left(t_{1}\right), \bar{f}\left(t_{2}\right), \ldots, \bar{f}\left(t_{N}\right)\right) \text { with } k \leqslant N
$$

Once the nominal value of the simulation result is known, the uncertainty associated with this result can be calculated by obtaining the sensitivities associated with each argument using a first-order sensitivity analysis or truncated Taylor Series approximation ((i.e. partial derivative of $x_{k}$ with respect to each argument)) shown in Eq. (4).

$$
\left(S_{X_{i}}\right)_{k}=\frac{\partial x_{k}}{\partial \bar{X}_{i}} \text { with } k=1,2, \ldots, N
$$

Note that, for this example,

$$
\frac{\partial x_{k}}{\partial f\left(t_{j}\right)}=0 \text { if } j \geqslant k
$$

because the sensitivity of the current position in time to forces from the future is equal to zero. Equation (5) is known as a causality condition (i.e. the future cannot affect the present).

Although feasible for this particular problem, in practice, obtaining explicit analytical expressions for $x_{k}$ in terms of all the arguments may not be possible for real world examples where source code availability is limited (e.g. FEA codes). Nevertheless, the first-order sensitivity coefficients can still be obtained by perturbing each of the arguments of $x_{k}$ as in this section. A numerical algorithm such as the Runge-Kutta method can then be used to solve Eq. (2) given the model parameters, the initial conditions, and the history of external inputs. The outcome of the numerical solution using the nominal parameters is a sequence of nominal results (Eq. (3)). The input parameters used for this example are presented in Table 1.

For this simulation, the total number of time-steps is $N=100$, over a 10 s duration. Therefore, the time-step is equal to $0.1 \mathrm{~s}$. The nominal simulation results (displacement) are displayed in Fig. 2 below.

A quick time invariance check can be performed by adding a perturbation to the external input force at different times and executing simulation runs for each perturbation. Furthermore, the nominal solution can then be subtracted from these simulations and the results superimposed (i.e. offset the time scale so that the simulation results are superimposed). In Fig. 3, a unit impulse perturbation was applied at $t=0.5 \mathrm{sec}$ and $t=1.0 \mathrm{sec}$. The nominal simulation results were then subtracted from the simulation results associated with each of these perturbations, and the time was offset in order to superimpose the results. These results are illustrated in Fig. 3 below.

As shown in Fig. 3, time invariance is an excellent assumption for this simple SDOF example. 
Table 1

SDOF system arguments and uncertainties

\begin{tabular}{lccc}
\hline Model parameter & Nominal value & $\begin{array}{c}\text { Standard } \\
\text { random } \\
\text { uncertainty }\end{array}$ & $\begin{array}{c}\text { Standard } \\
\text { systematic } \\
\text { uncertainty }\end{array}$ \\
\hline mass $(m)$ & $5 \mathrm{~kg}$ & - & $0.025 m$ \\
stiffness $(k)$ & $7 \mathrm{~N} / \mathrm{m}$ & - & $0.015 k$ \\
damping coefficient $(b)$ & $4 \mathrm{Ns}^{-1} \mathrm{~m}^{-1}$ & - & $0.025 b$ \\
Initial condition & - & - & - \\
displacement $\left(x_{0}\right)$ & $-0.2 \mathrm{~m}$ & - & $0.015 x_{0}$ \\
velocity $\left(v_{0}\right)$ & $-1 \mathrm{~ms}^{-1}$ & 3 & $0.02 v_{0}$ \\
Time-varying external input & & & 1 \\
forcing function $(f(t))$ & {$\left[100 \sin \left(2 \pi \frac{t_{k}}{\mathrm{sec}}\right) e \frac{-t_{k}}{\mathrm{sec}}\right] N$} & & \\
\hline
\end{tabular}

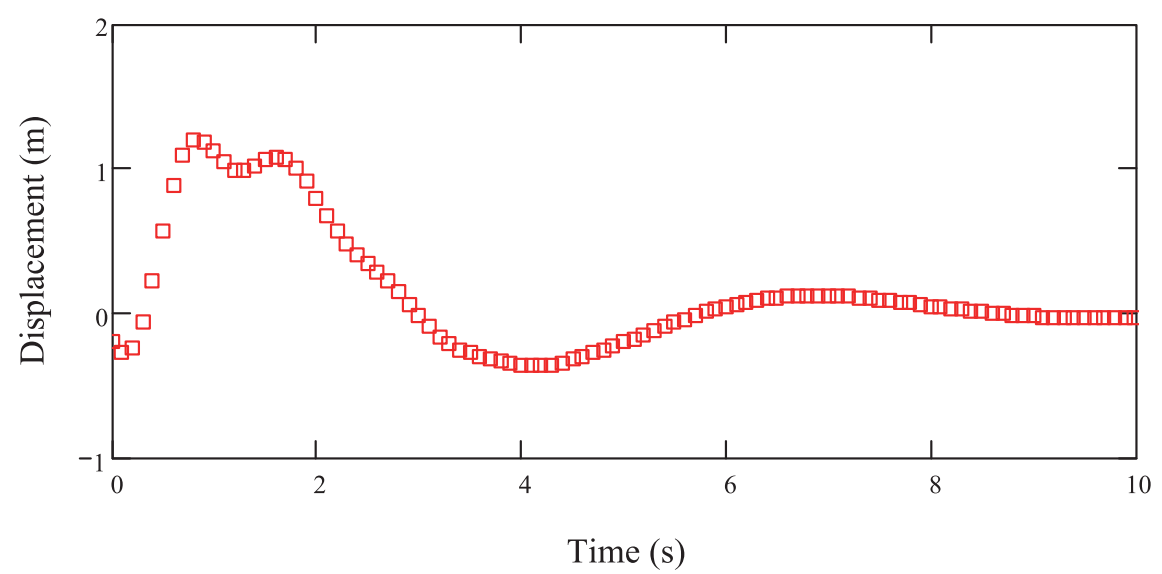

Fig. 2. SDOF system time-varying displacement resultss.

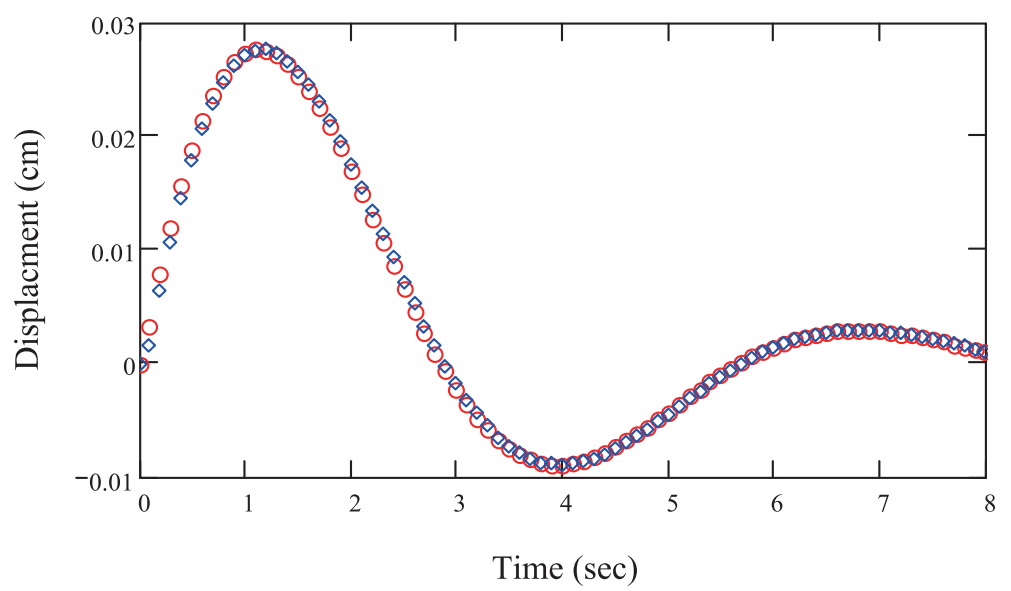

Fig. 3. Time invariance check.

The sensitivity of displacement with respect to changes in the mass $(m)$ can be obtained by running another numerical simulation that uses a slightly perturbed mass value, but otherwise identical parameters. The new differential equation to be solved is shown in Eq. (6).

$$
(m+\Delta m) \ddot{x}+b \dot{x}+k x=f(t)
$$

The solution of this mass-perturbed model can be labeled as follows: 


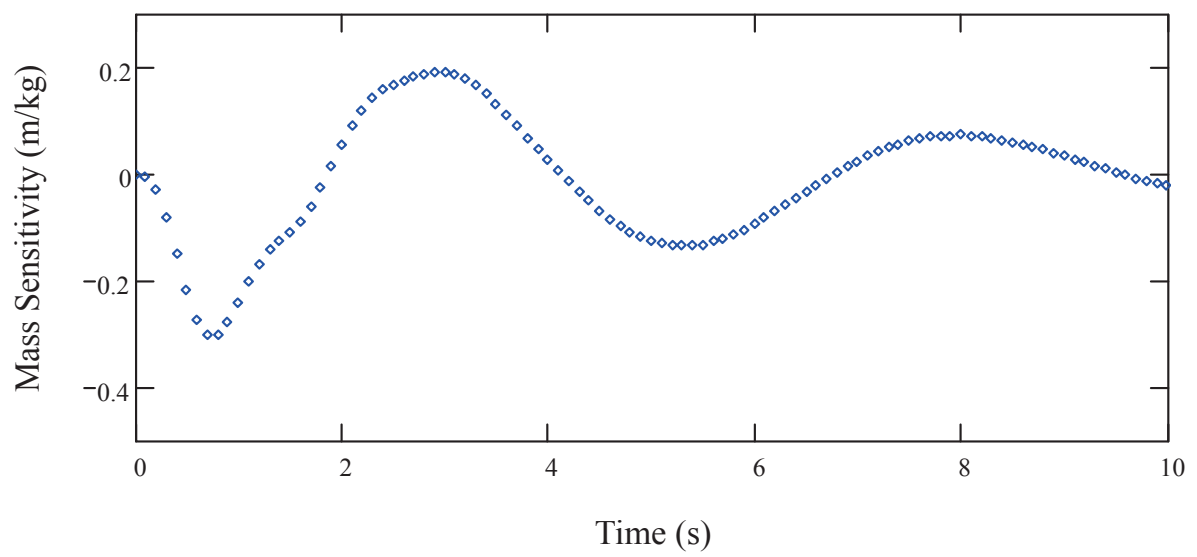

Fig. 4. Model sensitivity to mass perturbation.

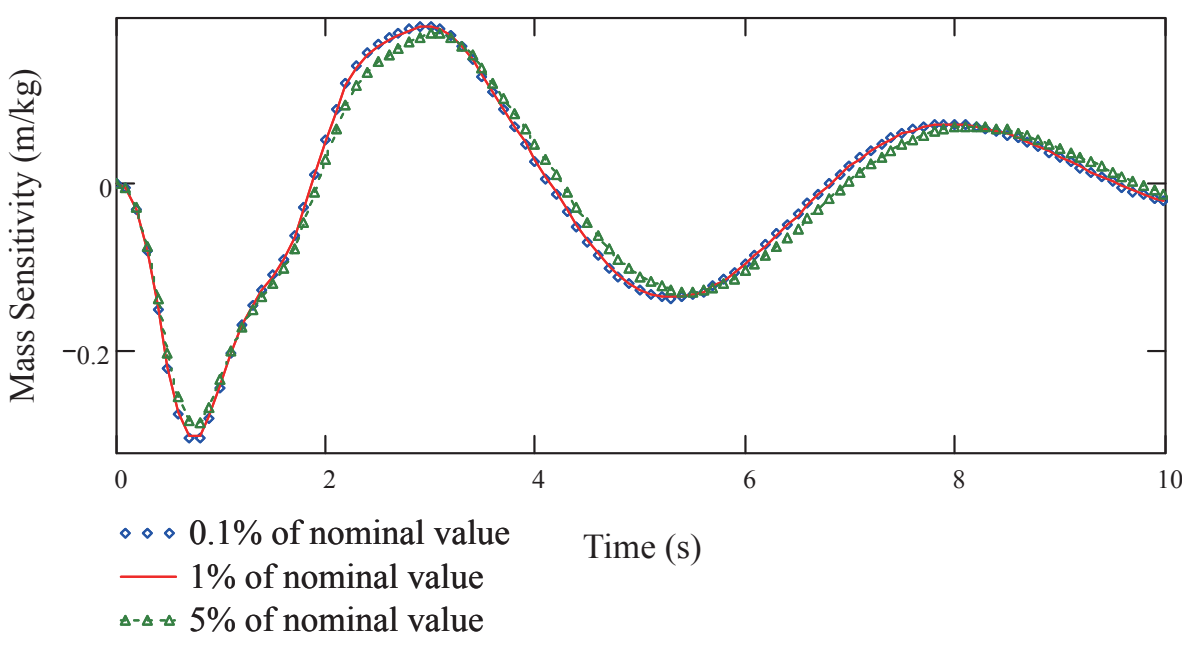

Fig. 5. Mass sensitivity convergence plot.

$$
x_{k}\left(\bar{k}, \bar{b}, \bar{m}+\Delta m, \bar{x}_{0}, \bar{v}_{0}, \bar{f}\left(t_{1}\right), \bar{f}\left(t_{2}\right), \ldots, \bar{f}\left(t_{N}\right)\right) \text { for } k=1,2, \ldots, 100
$$

The sensitivity at time $t_{k}$ due to small perturbations in the mass value can then be expressed as shown in Eq. (8). As stated earlier, Eq. (8) can be derived using a truncated Taylor Series approximation of the model response with respect to the model arguments.

$$
\left(S_{m}\right)_{k}=\frac{\partial x_{k}}{\partial X_{i}} \cong \frac{x_{k}\left(\bar{k}, \bar{b}, \bar{m}+\Delta m, \bar{x}_{0}, \bar{v}_{0}, \bar{f}\left(t_{1}\right), \bar{f}\left(t_{2}\right), \ldots, \bar{f}\left(t_{N}\right)-R_{k}\right.}{\Delta m} \text { for } k=1,2, \ldots, 100
$$

In Fig. 4, a plot of $\left(S_{m}\right)_{k}$ using SI units for $k=1,2, \ldots, 100$ using a value of $\Delta m=0.01 \bar{m}$.

Figure 5 shows how the sensitivity coefficients for perturbations in the mass converge as the perturbation $\Delta m$ is diminished from $5 \%$ to $0.1 \%$ of the nominal value, $\bar{m}$, of the mass. Since this is a first-order approximation of the sensitivity coefficient, we are essentially testing to determine whether the change in $x_{k}$ with respect to small changes in $\bar{m}$ remains constant for various values of $\Delta m$.

As shown in Fig. 5, the mass sensitivity remains approximately constant within the uncertainty in the mass, $\Delta m=0.01 \bar{m}$. This procedure is used to find the sensitivities due to the initial conditions, the damping, and the stiffness. Figures 6 and 7 show the sensitivity coefficients for perturbations in $b, k, x_{0}$, and $v_{0}$ (all in SI units).

The sensitivity coefficients due to the external inputs can be found in a similar manner as the model parameters and initial conditions. 


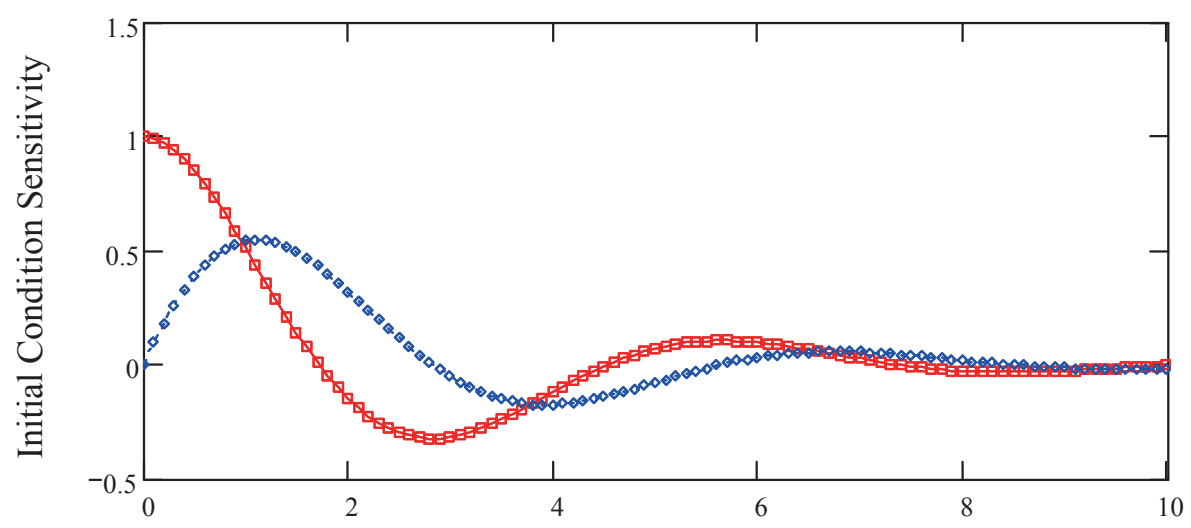

Time (s)

displacment sensitivity

$\diamond-\diamond$ velocity sensitivity

Fig. 6. Model sensitivity to perturbations in initial conditions.

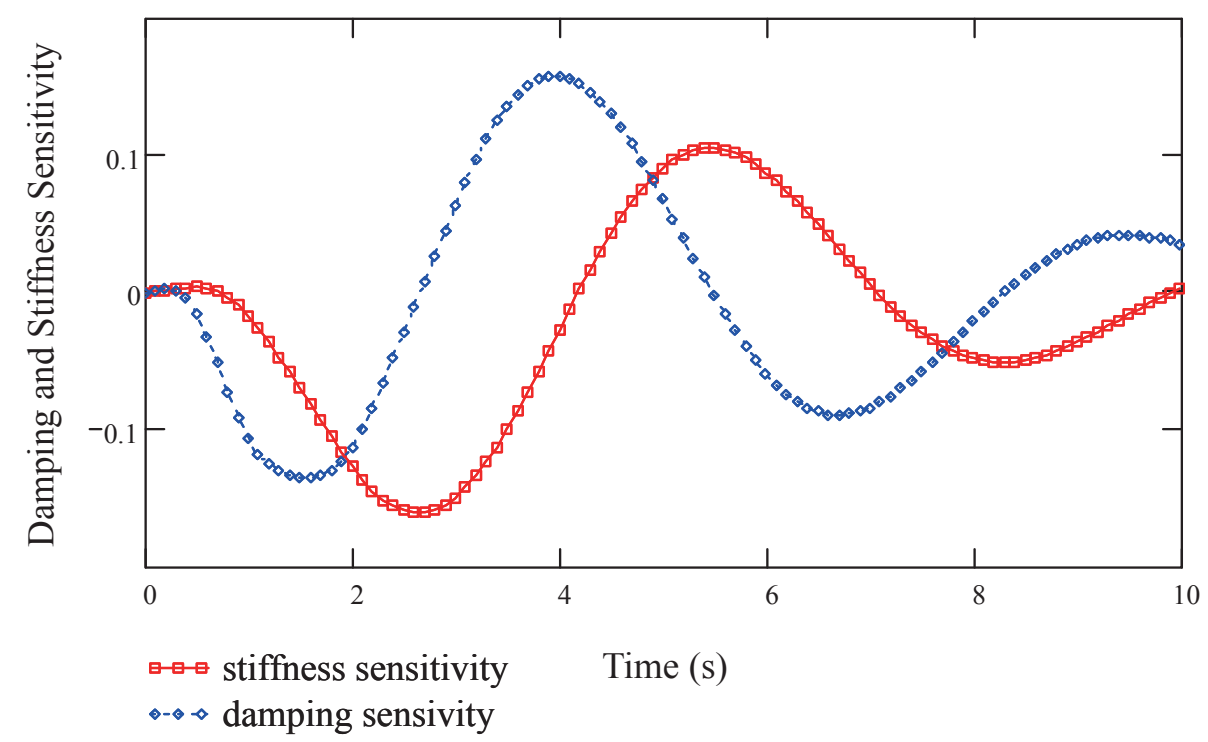

Fig. 7. Model sensitivity to perturbations in damping and stiffness coefficients.

$$
\left(S_{f_{1}}\right)_{k}=\frac{\partial x_{k}}{\partial f\left(t_{1}\right)} \quad, \quad\left(S_{f_{2}}\right)_{k}=\frac{\partial x_{k}}{\partial f\left(t_{2}\right)}, \ldots,\left(S_{f_{N}}\right)_{k}=\frac{\partial x_{k}}{\partial f\left(t_{N}\right)}
$$

For this example, the perturbation in the displacement with respect to small perturbations in the input force can be expressed as

$$
\hat{x}_{k}\left(\Delta \bar{f}\left(t_{1}\right), \ldots, \Delta \bar{f}\left(t_{N}\right)\right)=x_{k}\left(\bar{k}, \bar{b}, \bar{m}, \bar{x}_{0}, \bar{v}_{0}, \bar{f}\left(t_{1}\right)+\Delta f\left(t_{1}\right), \ldots, \bar{f}\left(t_{N}\right)+\Delta f\left(t_{N}\right)\right)-R_{k}
$$

The sensitivity coefficient to perturbations in can be obtained as follows

$$
\left(S_{f_{j}}\right)_{k}=\frac{\partial x_{k}}{\partial f\left(t_{j}\right)}=\frac{\hat{x}_{k}\left(0, \ldots, \Delta f\left(t_{j}\right), \ldots, 0\right)}{\Delta f\left(t_{j}\right)}
$$

Note that the only nonzero input perturbation is $\Delta f\left(t_{j}\right)$. All other external input perturbations are set to zero. Finding approximations to these sensitivities using the same numerical procedure used to find the sensitivities for $b$, 


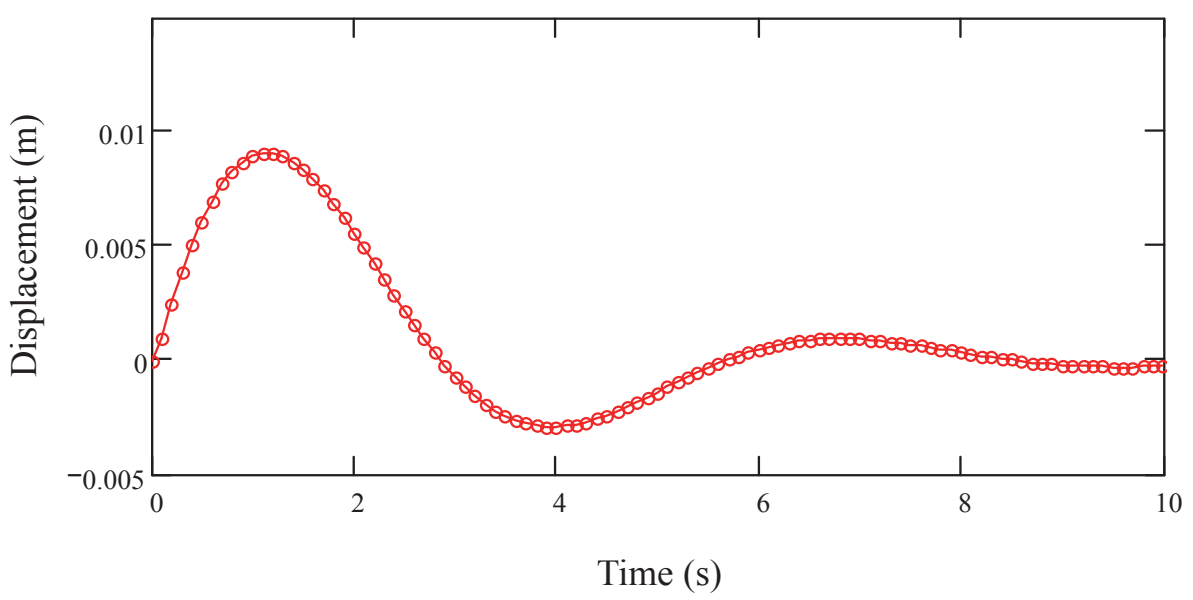

Fig. 8. Impulse response of SDOF system.

$k, x_{0}$, and $v_{0}$ requires at least 200 numerical simulations (i.e. two for each $f_{k}$ with $k=1,2, \ldots, 100$ ). The concept of superposition can be used to drastically reduce the number of simulations needed to calculate the sensitivities associated with the external input.

As stated earlier, linearity implies superposition of the input force. First-order sensitivity analysis applies to the region where the equations behave linearly with respect to small changes in the arguments of interest. Thus, one starts by assuming that the resultant displacement behaves linearly with respect to small changes in the input force. For this example, the output displacement is already a linear function of the input force. However, it is possible to obtain a linearized model, i.e., a first-order Taylor series approximation, for many nonlinear models using the process outlined below based on the discrete version of the Convolution integral. The Convolution integral, also known as Duhamel's Integral, is used to obtain the response of a linear dynamical system to arbitrary inputs by using the concept of superposition. Assuming a linear response to perturbations in the input, the discretized version of Duhamel's integral is simply

$$
\hat{x}_{k}\left(\Delta f\left(t_{1}\right), \ldots, \Delta f\left(t_{N}\right)\right)=\sum_{i=1}^{k} h_{k-i} \Delta f\left(t_{i}\right)
$$

where the sequence $\left\{h_{k}\right\}$, for $k=1,2, \ldots, N$, is known as the "impulse response" and is a special case of the Green's functions typically used to solve conductive heat transfer problems [5]. The sensitivity coefficient for the external input $f\left(t_{j}\right)$ is obtained by setting $\Delta f\left(t_{i}\right)=0$ for $i \neq j$ in Eq. (12) to obtain the following relationship:

$$
\left(S_{f_{j}}\right)_{k}=\frac{\partial x_{k}}{\partial f\left(t_{j}\right)} \cong \frac{\hat{x}_{k}\left(0, \ldots, \Delta f\left(t_{j}\right), \ldots, 0\right)}{\Delta f\left(t_{j}\right)}=h_{k-j} \text { for } \begin{gathered}
k \geqslant j \\
0, \text { otherwise }
\end{gathered}
$$

This shows that the sensitivity coefficients to the input force can be obtained from the coefficients of the impulse response, $h_{k}$. For instance, it is only necessary to calculate

$$
\left(S_{f_{1}}\right)_{k}=h_{k-1} \text { for } k=1,2, \ldots, N
$$

by using Eqs (12) and (13) to obtain Eq. (15) below.

$$
\left(S_{f_{1}}\right)_{k}=\frac{\partial x_{k}}{\partial f\left(t_{1}\right)} \cong \frac{x_{k}\left(\bar{k}, \bar{b}, \bar{m}, \bar{x}_{0}, \bar{v}_{0}, \bar{f}\left(t_{1}\right)+\Delta f\left(t_{1}\right), \bar{f}\left(t_{2}\right), \ldots, \bar{f}\left(t_{N}\right)-R_{k}\right.}{\Delta f\left(t_{1}\right)}
$$

Figure 8 presents a plot of the impulse response $h_{k}$ obtained by setting $\Delta f\left(t_{1}\right)=1$ and all other $\Delta f$ equal to zero. Note that, for this example, the choice of $\Delta f\left(t_{1}\right)$ is not critical as the differential equation is known to be linear with respect to the input force; thus, for a given $\Delta f\left(t_{1}\right)$, the response can be normalized by dividing it by $\Delta f\left(t_{1}\right)$.

Note that the impulse response damps down to zero. In many applications, the impulse response becomes negligible in a finite amount of time; truncating the negligible values results in a finite impulse response (FIR) model. 
The importance of the FIR model is that many of the sensitivity values become negligibly small and can be ignored in the uncertainty calculations.

The first-order Taylor Series approximation of the perturbed result is given in Eq. (16).

$$
\begin{aligned}
& x_{k}\left(\bar{k}, \bar{b}, \bar{m}, \bar{x}_{0}, \bar{v}_{0}, \bar{f}\left(t_{1}\right)+\Delta f\left(t_{1}\right), \ldots, \bar{f}\left(t_{N}\right)+\Delta f\left(t_{N}\right)\right) \cong R_{k}+\left(S_{k}\right)_{k} \Delta k \ldots \\
& \quad+\left(S_{b}\right)_{k} \Delta b+\left(S_{m}\right)_{k} \Delta m+\left(S_{x_{0}}\right)_{k} \Delta x_{0}+\left(S_{v_{0}}\right)_{k} \Delta v+\sum_{j=1}^{k} h_{k-j} \Delta f\left(t_{j}\right)
\end{aligned}
$$

Note that last term in the approximation Eq. (16) is obtained with the following substitution based on the derivation using the Convolution integral:

$$
\sum_{j=1}^{k}\left(S_{f_{j}}\right)_{k} \Delta f\left(t_{j}\right)=\sum_{j=1}^{k} h_{k-j} \Delta f\left(t_{j}\right)
$$

The combined uncertainty in the displacement is then derived using the expected value operation on Eq. (16) being mindful that the systematic and random errors associated with the external inputs, model parameter errors, and initial condition errors are assumed to be uncorrelated. Also, $\Delta k, \Delta b, \Delta m, \Delta x_{0}$, and $\Delta v_{0}$ are assumed to be due to systematic errors while $\Delta f_{k}$ is composed of random $(r)$ and systematic $(s)$ errors. Finally, the random errors associated with the external inputs are assumed to be uncorrelated (between time-steps) and identically distributed at each time-step. This total displacement uncertainty expression is presented as Eq. (18).

$$
\begin{gathered}
\left(u_{x}\right)_{k}^{2}=\left(S_{k}\right)_{k}^{2} u_{k}^{2}+\left(S_{b}\right)_{k}^{2} u_{b}^{2}+\left(S_{m}\right)_{k}^{2} u_{m}^{2}+\left(S_{x_{0}}\right)_{k}^{2} u_{x_{0}}^{2}+\left(S_{v_{0}}\right)_{k}^{2} u_{v_{0}}^{2}+\ldots \\
\left(\sum_{j=1}^{k} h_{k-j}^{2}\right) u_{f_{r}}^{2}+\left(\sum_{j=1}^{k} h_{k-j}\right)^{2} u_{f_{s}}^{2}
\end{gathered}
$$

The last two terms in Eq. (18) are derived using the following expression for the error in the external input and making the appropriate substitutions as shown in Eqs (19) and (20).

$$
\begin{aligned}
& \Delta f\left(t_{j}\right)=\Delta f_{s}+\left(\Delta f_{r}\right)_{j} \\
& \sum_{j=1}^{k}\left[h_{k-j} \Delta f\left(t_{j}\right)\right]=\sum_{j=1}^{k} h_{k-j}\left[\Delta f_{s}+\left(\Delta f_{r}\right)_{j}\right]=\left(\sum_{j=1}^{k} h_{k-j}\right)\left(\Delta f_{s}\right)+\sum_{j=1}^{k}\left[h_{k-j}\left(\Delta f_{r}\right)_{j}\right]
\end{aligned}
$$

After taking the expected value of the resulting two terms in the simplified result of Eq. (20), the following expanded set of equations representing the last two terms in Eq. (18) is obtained for time-steps $k=1,2, \ldots, N$ :

$$
\begin{aligned}
& E\left\{\sum_{j=1}^{1}\left[h_{1-j} \Delta f\left(t_{j}\right)\right]\right\}=\left(h_{0}\right)^{2}\left(u_{f_{s}}\right)^{2}+\left(h_{0}\right)^{2}\left(u_{f_{r}}\right)^{2} \\
& E\left\{\sum_{j=1}^{2}\left[h_{2-j} \Delta f\left(t_{j}\right)\right]\right\}=\left(h_{1}+h_{0}\right)^{2}\left(u_{f_{s}}\right)^{2}+\left[\left(h_{1}\right)^{2}\left(u_{f_{r}}\right)^{2}+\left(h_{0}\right)^{2}\left(u_{f_{r}}\right)^{2}\right] \\
& \vdots \\
& E\left\{\sum_{j=1}^{N}\left[h_{N-j} \Delta f\left(t_{j}\right)\right]\right\}=\left(h_{N-1}+h_{N-2}+\ldots+h_{0}\right)^{2}\left(u_{f_{s}}\right)^{2}+ \\
& {\left[\left(h_{N-1}\right)^{2}\left(u_{f_{r}}\right)^{2}+\left(h_{N-2}\right)^{2}\left(u_{f_{r}}\right)^{2}+\ldots+\left(h_{0}\right)^{2}\left(u_{f_{r}}\right)^{2}\right]}
\end{aligned}
$$




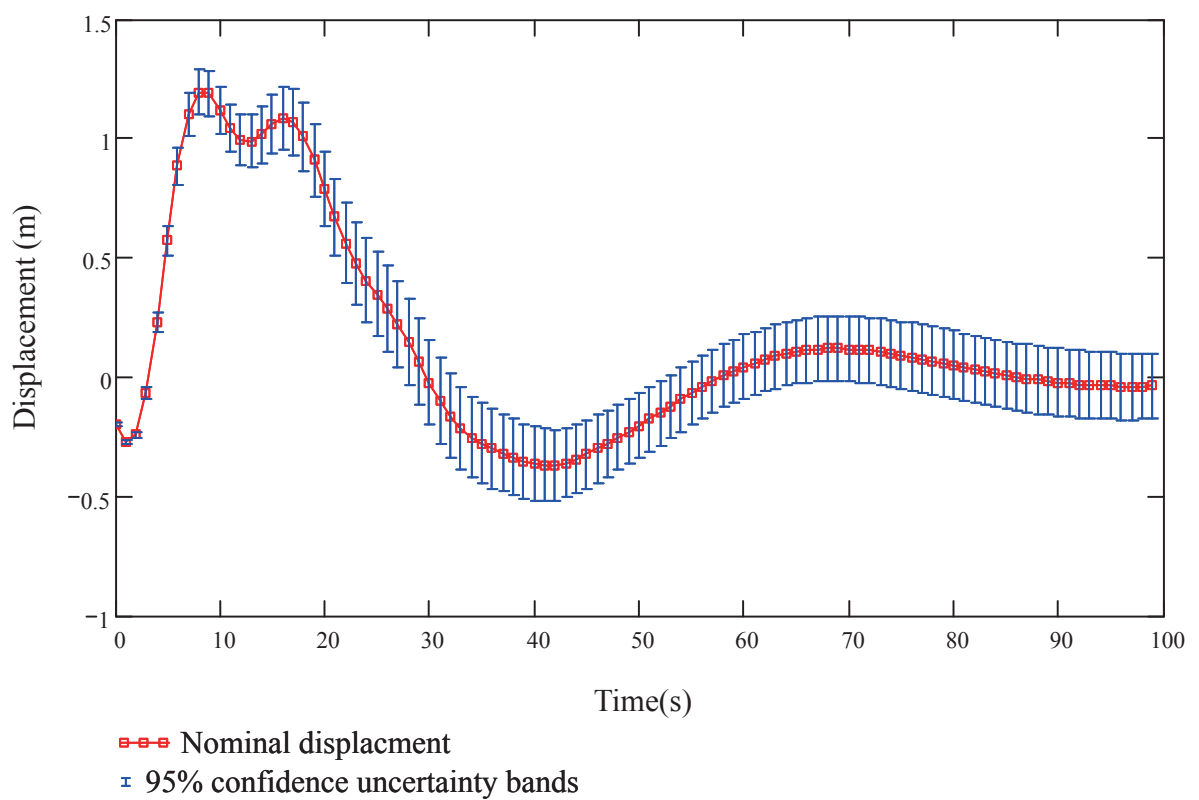

Fig. 9. 95\% confidence displacement uncertainty bands.

After simplification of Eq. (21), the last two terms in Eq. (18) are obtained. Finally, the 95\% confidence interval, assuming a normally distributed set of simulation results, is approximated using Eq. (22).

$$
x_{k}=R_{k} \pm 2\left(u_{x}\right)_{k}
$$

Figure 9 shows the $95 \%$ confidence upper and lower uncertainty bounds for the displacement results calculated using Eqs (18) and (22). Note that Fig. 9 illustrates the uncertainty band associated with each simulation realization.

In the following section, a practical example using commercially available FEA software is presented.

\section{Case study}

In this section, a realistic FEA example is provided to illustrate the impact of this type of transient uncertainty analysis methodology for real world time-varying engineering applications. A schematic of the system of interest is illustrated in Fig. 10.

Figure 10 illustrates a system with a piece of shock-hardened equipment which is isolated by isolation mounts on a primary structure that is also isolated. The success of this design effort hinges on the ability of the system to withstand shock loading, reduce the equipment acoustic footprint, and limit shock excursions. However, for this particular case, the excursions resulting from a shock load are of particular interest since the space available for the system is extremely limited. In the following sections, the model used to represent the system illustrated in Fig. 10 is briefly discussed. Note that the physical fidelity of this model is second to the main purpose of this paper which is to show the applicability of this type of uncertainty methodology for real engineering applications.

\subsection{Model attributes and results}

The system shown in Fig. 10 was modeled using commercially available FEA software to determine the amount of shock excursion experienced by the double isolated piece of equipment due to a time-varying external input. The external input data is an experimentally derived acceleration time history which is input below the primary shock mounts in increments of $0.001 \mathrm{sec}$ over a time period of $1 \mathrm{sec}$.

The model used to represent the system given in Fig. 10 was constructed using six degree-of-freedom (DOF) nodes and spring/damper elements, plate elements, rigid elements and lumped mass elements for a total of 1723 
Table 2

FEA case study model arguments and standard uncertainties

\begin{tabular}{lccc}
\hline Model parameter & Nominal value & $\begin{array}{c}\text { Standard } \\
\text { random } \\
\text { uncertainty }\end{array}$ & $\begin{array}{c}\text { Standard } \\
\text { systematic } \\
\text { uncertainty }\end{array}$ \\
\hline Primary mount stiffness (vertical), $(\mathrm{kN} / \mathrm{m})$ & 2754 & - & 30 \\
Secondary mount stiffness (vertical), $(\mathrm{kN} / \mathrm{m})$ & 1057 & - & 11 \\
Primary mount stiffness (transverse), $(\mathrm{kN} / \mathrm{m})$ & 573 & - & 17 \\
Secondary mount stiffness (transverse), $(\mathrm{kN} / \mathrm{m})$ & 248 & - & 6 \\
Secondary mount damping, $(\mathrm{kN}-\mathrm{sec} / \mathrm{m})$ & 2.8 & - & 0.7 \\
Primary mount damping, $(\mathrm{kN}-\mathrm{sec} / \mathrm{m})$ & 4.2 & - & 1.0 \\
Beam web thickness, $(\mathrm{cm})$ & 0.584 & - & 0.013 \\
Beam flange thickness, $(\mathrm{cm})$ & 0.711 & - & 0.013 \\
Equipment CG mass, $(\mathrm{kg})$ & 454 & - & 6.83 \\
Overall structural damping coefficient & 0.1 & - & 0.025 \\
Time-varying External Input & & & $0.1 \%$ \\
Acceleration Input, $a(t)$ & Time-varying & $5 \%$ & \\
\hline
\end{tabular}

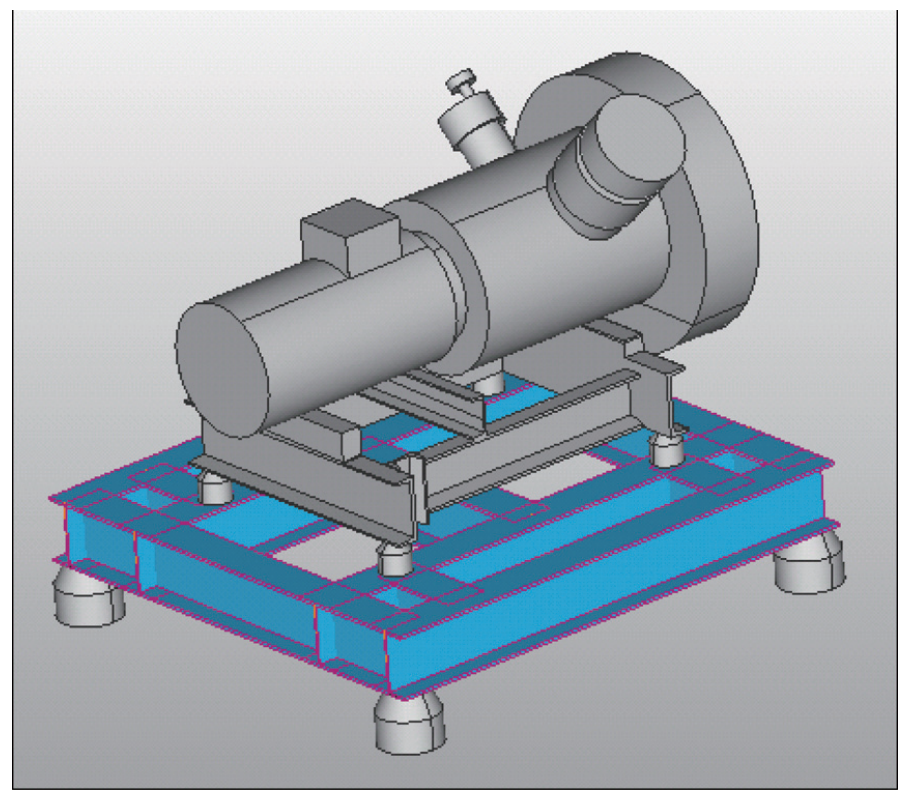

Fig. 10. Double-isolated equipment (air compressor).

nodes (10344 DOF) and 1595 elements. In addition, average time needed to perform the computations for a single simulation is $\sim 3.2 \mathrm{~min}$. Figure 11 presents the finite element model (FEM) of the system with exaggerated nodal deformations which is included only to provide a snapshot of what the analyst would typically see during each frame of a simulation.

The significant model parameters influencing the shock excursion including nominal values and standard uncertainties are presented in Table 2. All significant modeling parameters were either measured, obtained from vendor data or estimated using past experience and/or engineering judgment.

Of course, there exists a possibility that other model parameters contribute to the uncertainty in the simulation results (i.e. material properties, structural geometry, etc...). However, for the purposes of this example, these parameters were assumed to provide negligible contributions to the uncertainty in the simulation results. Though outside of the scope of this paper, a full-scale validation exercise, e.g. see [6], could provide additional information associated with the validity of this assumption. Some additional assumptions include:

1) negligible material property effects due to high strain rate (i.e. static properties vs. dynamic properties)

2) linear elastic material properties in primary structure and shock mounts 


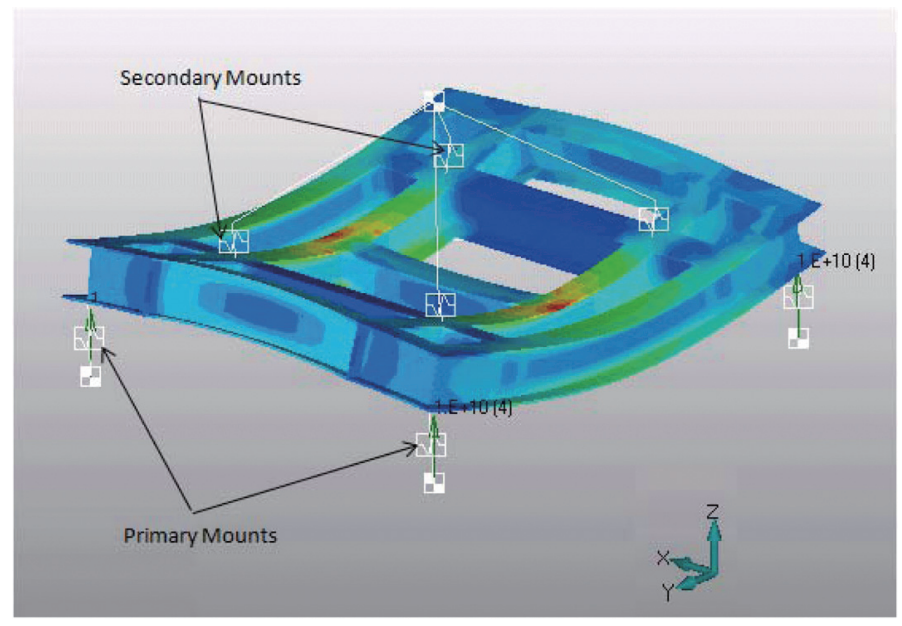

Fig. 11. System FEM.

3) small displacements in primary structure

4) negligible numerical and discretization error

The errors introduced due to the assumptions listed above can be lumped into an error due to modeling assumptions [7-9]. Typically, the modeler/experimentalist does not have information associated with this type of error $a$ priori; however, one can use quantitative validation methodology to estimate the uncertainty associated with this modeling assumption error. This type of effort includes comparing simulation results to benchmark experimental results/analysis (with associated uncertainties) to determine the validity of the modeling assumptions. However, for the purposes of this paper, the focus is primarily on the uncertainty associated with the simulation results.

As discussed earlier, the model response of interest is the shock excursion of the double isolated piece of shockhardened equipment. In addition, though outside the scope of this paper, one could just as easily monitor other time-varying parameters associated with the primary structure (e.g. stress, strain energy, dissipated energy etc....). The nominal model response due to the external input with the nominal parameters listed in Table 2 is presented in Fig. 12.

Notice that the total vertical displacement of the equipment CG exceeds the amount of possible displacement (equal to $\sim 17.75 \mathrm{~cm}$ ) which is generally limited by the bottoming of the mounts or simply the elastic limit of the isolation system. This unrealistic response is due to the modeling assumption of linear spring elements (i.e. linear load vs. displacement element attributes). Adding spring elements with nonlinear load vs. displacement curves and hysteresis effects would result in a more realistic simulation; however, since this example is not intended to be a model validation exercise, the current modeling assumptions remain unchanged for this exercise. During a model validation effort, these assumptions could be improved and the uncertainty analysis repeated to more accurately represent the physics of the problem [6].

The procedure outlined in the previous sections can now be used to calculate the sensitivities associated with each model parameter. Since there are 10 model parameters, a total of 20 simulation runs must be conducted (i.e. 2 for each parameter) to assure that first-order sensitivity coefficients are appropriate (i.e. truncated Taylor Series approximation is valid). This assumption is valid when approximately constant sensitivities exist within the uncertainty bands associated with each model argument. For this particular problem, the model parameter perturbations are set to the edges of the standard uncertainty bands for each parameter. Moreover, the combination of errors for each argument errors is assumed to be normally distributed which is naturally supported by the Central Limit Theorem for many engineering applications [2,10]. In Fig. 13, the sensitivities associated with the overall structural damping coefficient $(G)$ are examined as the overall structural damping coefficient perturbation is varied from $0.1 \pm 0.025$.

As illustrated in Fig. 13, the sensitivities of the shock excursion with respect to $G$ remain fairly constant for different perturbation magnitudes over the entire time span of interest. This fact is also true for the remaining model arguments presented in Table 2 . 


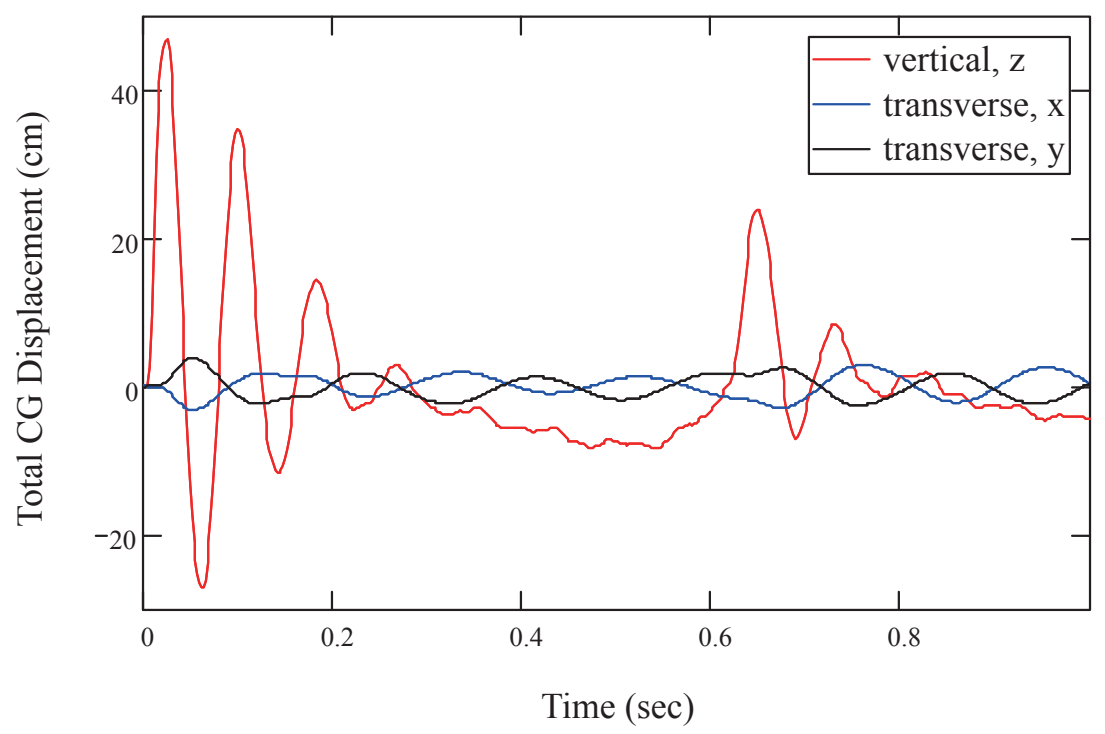

Fig. 12. Nominal simulation output.

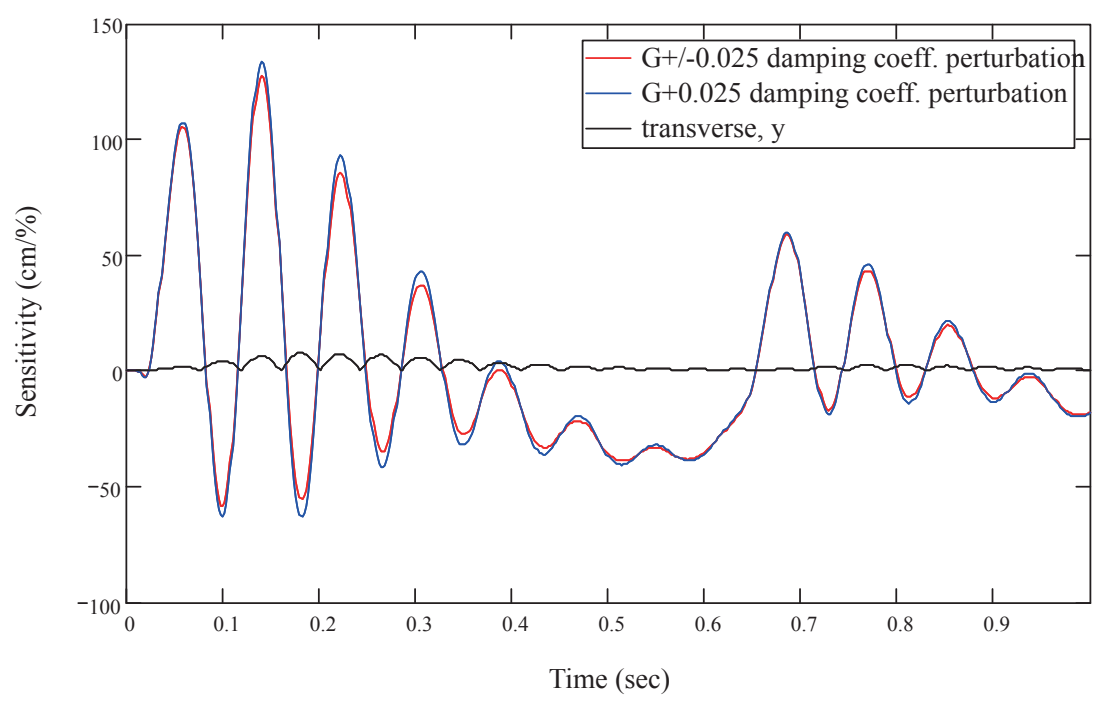

Fig. 13. Overall structural damping coefficient sensitivities.

After calculating first-order sensitivities associated with each model parameter, the sensitivities of the simulation results to perturbations in the external input can be calculated using the impulse response as described in the previous sections. Figure 14 provides the results of the impulse response of the system to a unit step input. As shown earlier, this response is used to calculate the sensitivities associated with the external input for all time-steps.

Finally, the total uncertainty in the result can be calculated in the same manner as the previous example using an equation of the form presented as Eq. (18). The total uncertainty in the vertical CG displacement is given in Fig. 15.

Notice that in Fig. 15, for the sake of simplicity, the uncertainty intervals were displayed as continuous curves about the nominal simulation results. However, these results are still to be interpreted as piecewise uncertainty bands about each simulation realization. For this particular example, the time-step resolution made illustrating uncertainty bounds for each simulation realization infeasible.

The results shown in Fig. 15 could facilitate a conservative excursion envelope for the piece of isolated equipment which could greatly impact the design of the associated equipment space and adjacent equipment spaces. In practice, 


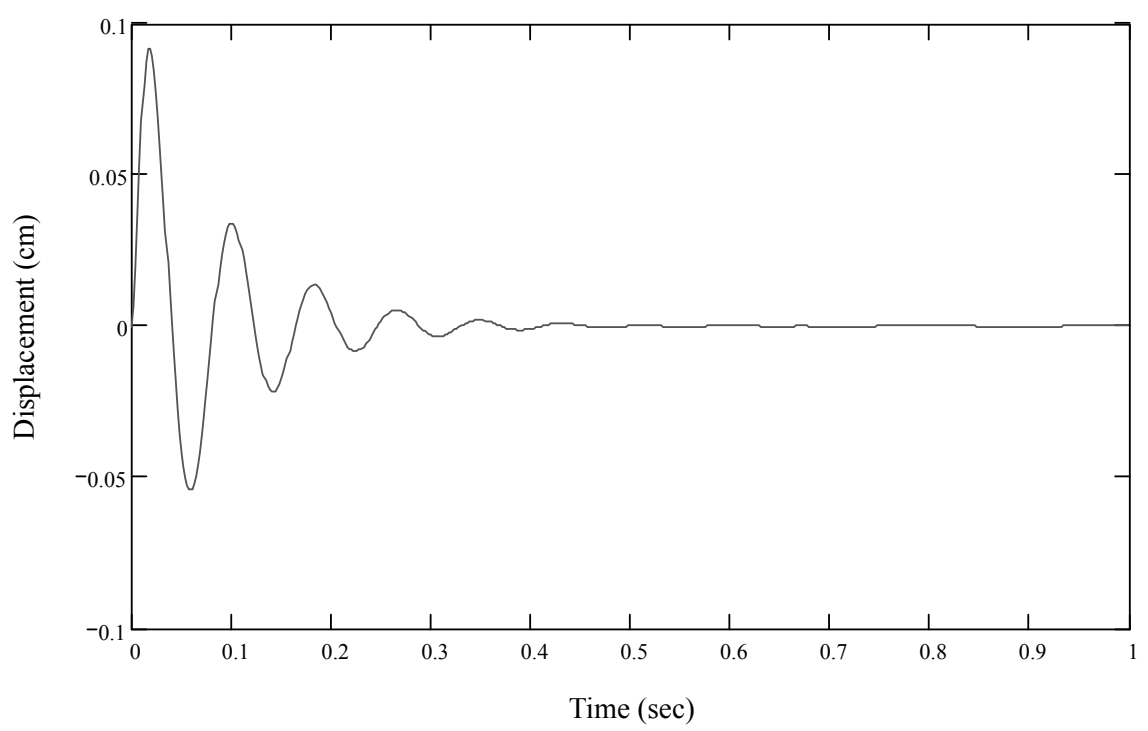

Fig. 14. Impulse response results.

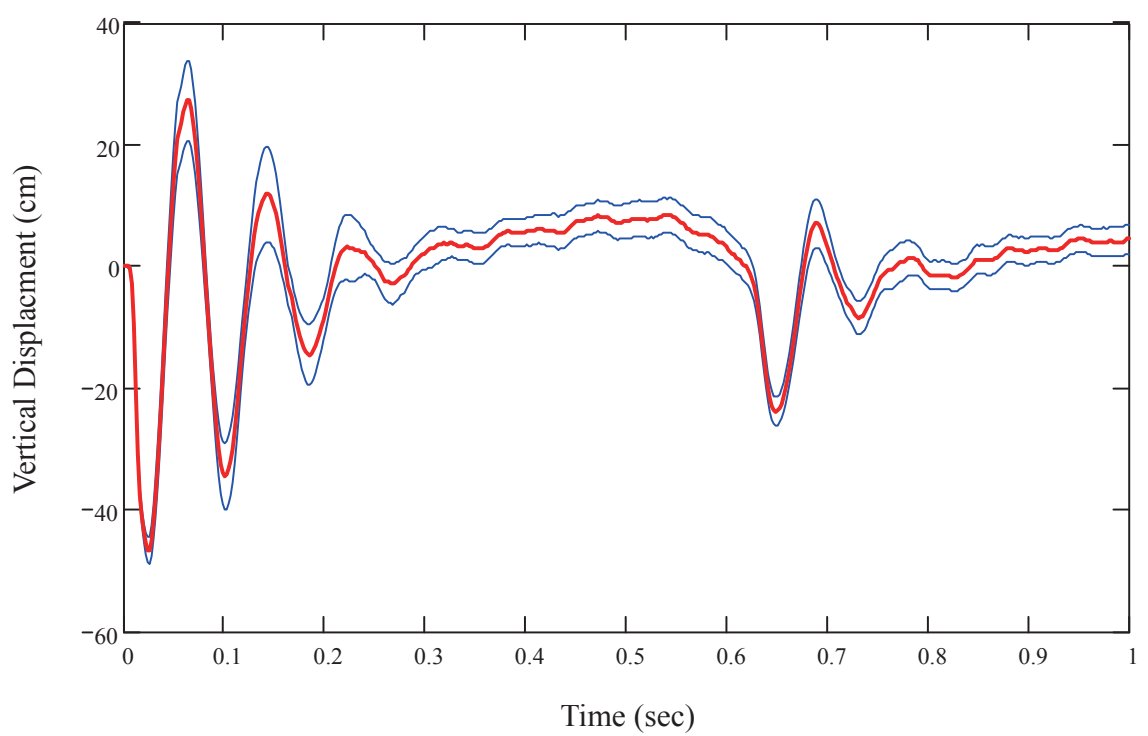

Fig. 15. Nominal simulation results with $95 \%$ confidence uncertainty bands.

provided the uncertainties are precisely formulated and understood, the 95\% uncertainty bands might serve as a strict "factor of safety". Whereas factors of safety are typically overly conservative and derived with predominantly engineering judgment, this level of understanding provides the support needed to determine a factor of safety which is less conservative while maintaining a level of comfort.

\section{Summary and conclusions}

In the previous sections, time-varying uncertainty methodology using the impulse response and convolution integral was introduced for LTI uncertainty models. Two examples were given to derive and show the advantages of 
the methodology particularly with respect to drastically reducing the number of simulation runs needed to resolve the uncertainty in a set of time-varying simulation results. These types of simulations are prevalent in the shock and vibration community; therefore, especially from a quantitative model validation standpoint, the ability to accurately determine the time-varying uncertainties associated with simulation results is crucial. Without this information, meaningful model validation becomes impossible.

In order to provide a real world example, a piece of double isolated equipment was modeled using commercially available FEA software. For this particular example, 21 simulation runs were needed to calculate the uncertainty associated with the simulation results. Two runs were needed for each model parameter, and one run was needed to obtain the impulse response to a unit step input. The total time needed for the 21 simulations runs is equal to $1.12 \mathrm{hrs}$. Using traditional uncertainty methodology, 2020 runs would be needed to resolve the uncertainty associated with the transient model response of interest. This includes the 20 runs for the model parameters, and a single run for each forcing function increment (i.e. 1 for each time-step) resulting in a total estimated computational time of 107.7 hrs. Therefore, using the methodology presented herein, the computation time needed to conduct the required model runs to resolve the simulation uncertainty is $\sim 1 \%$ of the time one would need using traditional methodology (i.e. calculating model sensitivities for each time-varying input at each time-step). Especially for models with lengthy run times, this analysis is extremely costly possibly making program objectives unattainable. An important note is that, while this methodology dramatically decreases the runs needed to resolve the time-varying uncertainties, even 20 simulation runs may prove too computationally expensive for many programs. However, history has shown that computational power doubles approximately every two years (Moore's Law). If this trend continues, this type of analysis could be feasible within the next 5-10 years for even the most complicated structural models.

\section{References}

[1] American National Standards Institute/American Society of Mechanical Engineers, Test Uncertainty, PTC 19.1-2005, ASME, New York, 2005.

[2] H.W. Coleman and W.G. Steele, Experimentation and Uncertainty Analysis for Engineers, John Wiley \& Sons, New York, 1999.

[3] C.M. Harris, Shock and Vibration Handbook, McGraw-Hill, Inc., New York, 1987.

[4] W.T. Thomson, Theory of Vibration with Applications, (2nd edition), Prentice-Hall Inc., NJ, 1981.

[5] J.N. Dewynne and J.M. Hill, Heat Conduction, Blackwell Scientific Publications, Boston, 1987.

[6] J.W. Weathers, R.P. Taylor and J.B. Weathers, CFD Simulation of a Meso-scale Combustor: An Exercise in Verification and Validation, Journal of Thermal Science and Engineering Applications 1(1) (2009).

[7] J.B. Weathers, R. Luck and J.W. Weathers, An exercise in model validation: Comparing univariate statistics and Monte Carlo-based multivariate statistics, Reliability Engineering and System Safety 94, 1695-1702.

[8] J.B. Weathers, R. Luck and J.W. Weathers, A Modular Approach to Linear Uncertainty Analysis, ISA Transactions 49(1) (2010), 19-26.

[9] H.W. Coleman and F. Stern, Uncertainties and CFD Code Validation, ASME J Fluids Eng 119 (1997), 795-803.

[10] L.L. Lapin, Probability and Statistics for Modern Engineering, Duxbury Press, California, 1990.

[11] J. Langston et al., Uncertainty Analysis for a Large-Scale Transient Simulation of a Notional All-Electric Ship Pulse Load Charging Scenario, 10th International Conference on Probabilistic Methods Applied to Power Systems (2008), 1-8.

[12] M.N. Avramova and K.N. Ivanov, Verification, validation and uncertainty quantification in multi-physics modeling for nuclear reactor design and safety analysis, Progress in Nuclear Energy 52(7) (2010), 601.

[13] M. Karkee and B.L. Steward, Local and global sensitivity analysis of a tractor and single axle grain cart dynamic system model, Biosystems Engineering 106(4) (2010), 352.

[14] Z.H. Cai, Y.W. Yang and Y. Liu, Dynamic response of structures with uncertain parameters, IOP Conference Series: Materials Science and Engineering 10(1) (2010). 

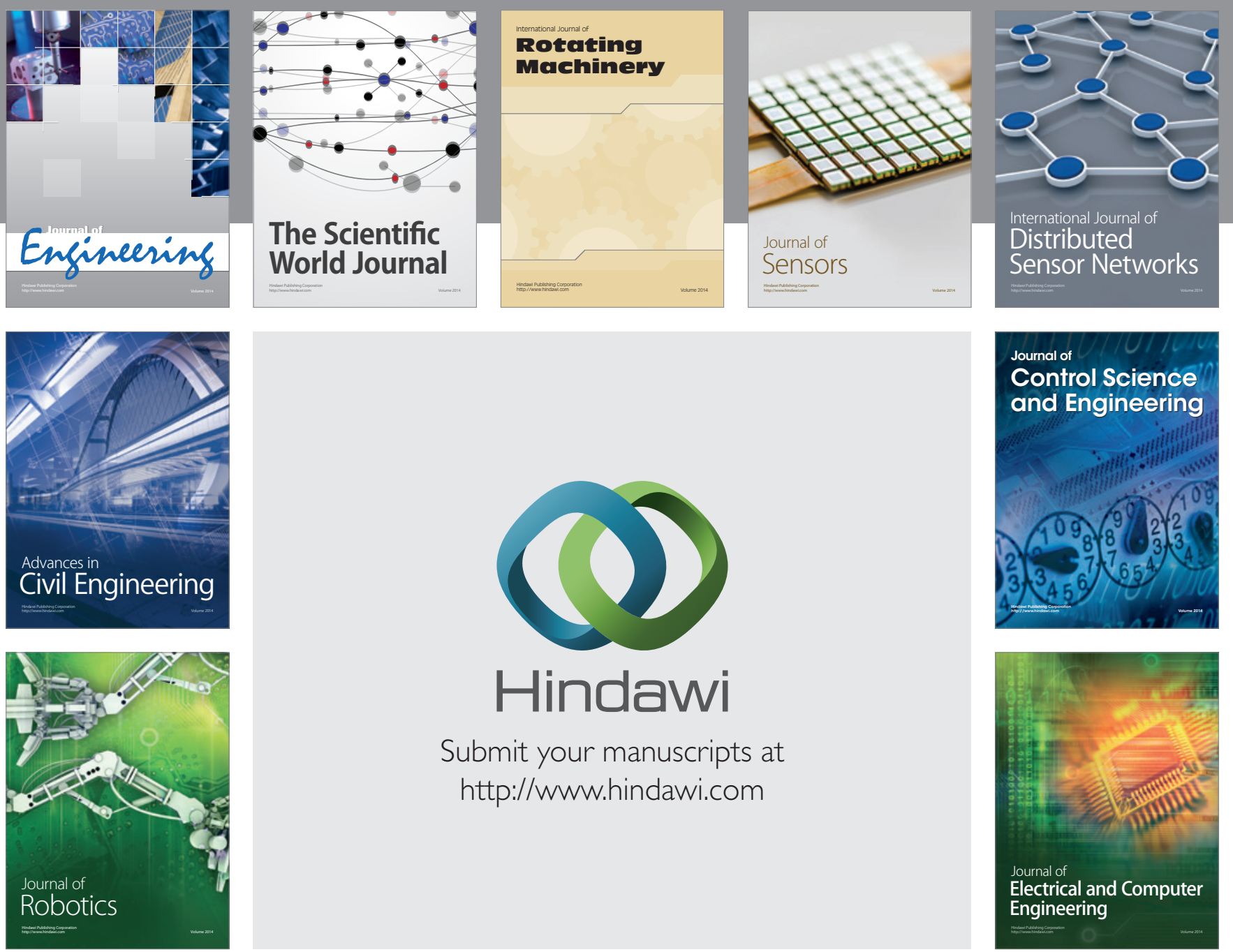

Submit your manuscripts at

http://www.hindawi.com
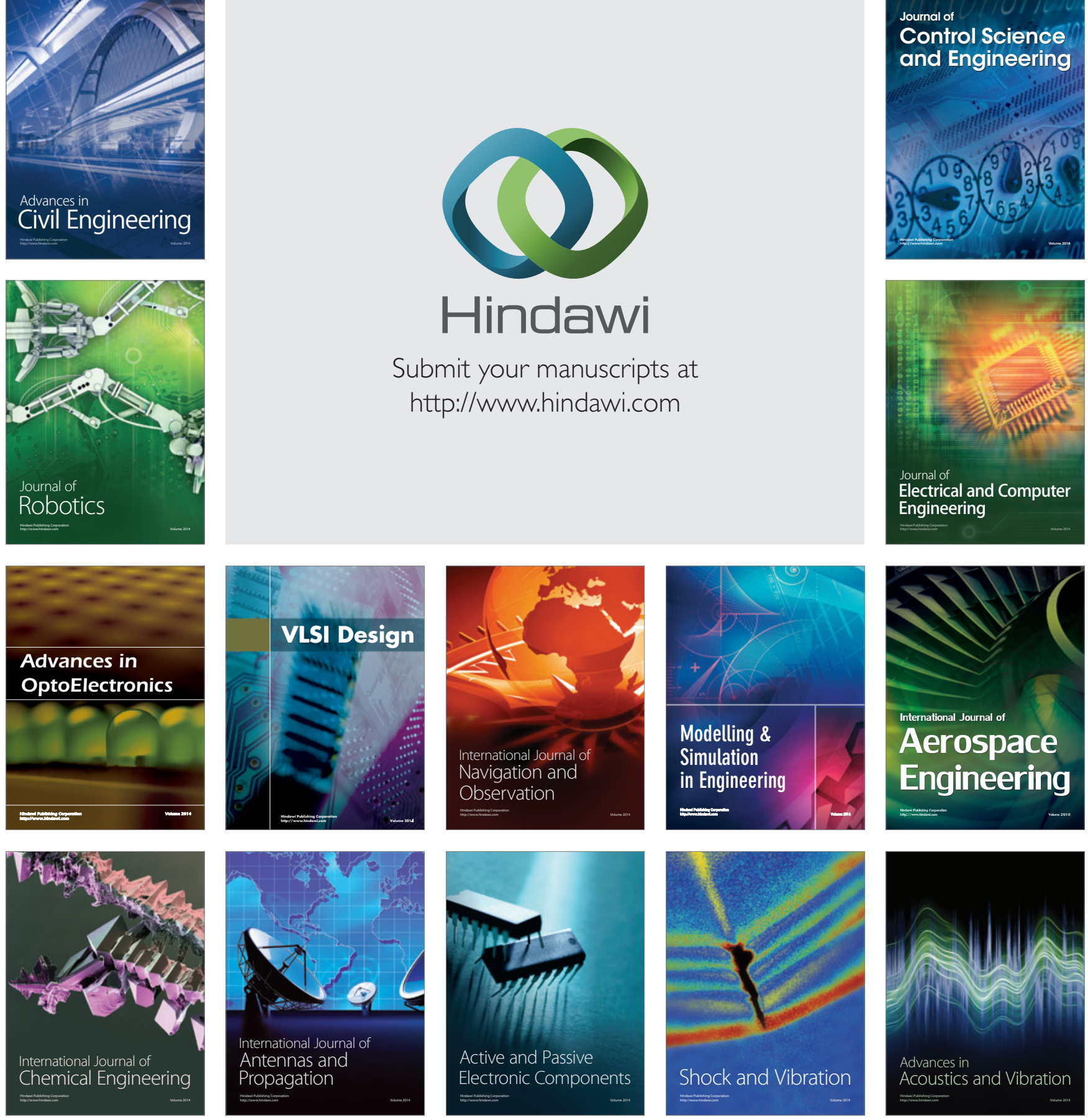